\title{
Detection of crop heights by UAVs based on the Adaptive Kalman Filter
}

\author{
Pingfan Xu, Hongchao Wang, Shenghui Yang, Yongjun Zheng* \\ (College of Engineering, China Agricultural University, Beijing, 100083, China)
}

\begin{abstract}
Detection of crop heights by UAVs is fast and accurate and can reflect the growth situation of crops. The core of this operation is the measurement of UAV flight altitude. In order to improve the accuracy of the response to the variation of flight altitude, a light-weight altitude detection system was developed for plant-protection UAVs. A millimeter wave radar (MWR) was used as the altitude detector. Moreover, a data fusion algorithm based on the Adaptive Kalman Filter (AKF) was developed. The altitude data by the MWR, the angle data by the Inertial Measurement Unit (IMU) and the position data by Global Positioning System (GPS) were processed by the AKF to obtain optimal updated values, and the optimal updated values was fused to establish the distribution map of crop heights. Results of the trials in the condition of the flight heights of $5 \mathrm{~m}$ and $10 \mathrm{~m}$ indicated that: 1) compared with the direct detection by the MWR, the error of detection was reduced by $0.035 \mathrm{~m}$. 2) compared with real crop heights, the error of detection was $0.02 \mathrm{~m}$. The developed system could achieve the accurate detection of the crop height, providing a new theoretical model and technical idea for the UAVs configured for plant-protection. Keywords: millimeter wave radar, Adaptive Kalman Filter, data fusion, altitude detection
\end{abstract} DOI: $10.33440 /$ j.ijpaa.20210401.166

Citation: Xu P F, Wang H C, Yang S H, Zheng Y J. Detection of crop heights by UAVs based on the Adaptive Kalman Filter. Int J Precis Agric Aviat, 2021; 4(1): 52-58.

\section{Introduction}

The measurement of crop height is of great significance to the normal growth of crops ${ }^{[1-8]}$. At present, plant protection UAV is widely applied in plant protection ${ }^{[9,10]}$. Detection of crop heights by UAVs is fast and accurate and can reflect the growth situation of crops. The core of this operation is the detection of flight altitude. Currently, there are many studies regarding the altitude detection for UAVs. Ultrasonic sensors ${ }^{[1]}$, $\operatorname{LIDAR}^{[12,13]}$, Image sensors ${ }^{[14]}$ and millimetre wave radar (MWR) ${ }^{[15-18]}$ are commonly used as measurement devices. Detection by a single sensor is a basic approach. For instance, Zhou et $\mathrm{al}^{[19]}$ utilised a type of high-accuracy MWR to measure the altitude of the UAV. The detection was accurate with less than $1 \%$ error in the condition of 15-meter-less flight, but the cost based on a single high-accurate MWR is very high. The accuracy of the measurement highly relies on that of the sensor.

In order to solve the shortcomings of single sensor detection, some researchers applied multi-sensor information fusion methods to fuse data from senso he Extended Kalman Filter (EKF). Based on this, Campos et al ${ }^{[24]}$ fused the data form a GPS, an IMU and an optical flow sensor to obtain estimated altitudes. The results illustrated that when the UAV flew at a constant speed and the flight altitude was $5 \mathrm{~m}$, the detection altitude error was $0.135 \mathrm{~m}$. However, if the drone accelerates, the detection accuracy fluctuates greatly. In order to improve the detection stability, Huang et $\mathrm{al}^{[25]}$ and $\mathrm{Tu}$ et $\mathrm{al}^{[26]}$ fused the data from a barometer, a GPS, and an

Received date: 2021-03-11 Accepted date: 2021-03-30

Biographies: Pingfan Xu, Master, research interests: Research on computer measurement and control technology, intelligent detection and imitating ground technology, Email: s20193071142@cau.edu.cn; Hongchao Wang, research interests: Research on computer measurement and control technology; Shenghui Yang, $\mathrm{PhD}$, research interests: computer measurement and control technology, Email: yang_sheng_h@126.com.

*Corresponding author: Yongjun Zheng, $\mathrm{PhD}$, Professor, research interests: intelligent detection for agriculture machine, Mailing Address: College of Engineering, China Agricultural University. Email: zyj@cau.edu.cn.
IMU, using an adaptive complementary filtering algorithm or Kalman filter for data filtering. The methods had high detection accuracy, but were not suitable for low altitude detection. Hu et $\mathrm{al}^{[27]}$ proposed a low altitude detection method, which filtered and fused the data of a barometer, an ultrasonic sensor, a GPS and an IMU by Fault Tolerant Kalman Filter (FTKFT). The maximum altitude error was $0.5 \mathrm{~m}$ when the UAV flying altitude was $2.5 \mathrm{~m}$. The error was a little large. Wu et $\mathrm{al}^{[28]}$ fused GPS data with the data of 3 MWRs by the Kalman Filter. The error of this method was low. However, in order to reduce the errors, too many sensors were applied and the amount of data processed was too large. Based on the idea of achieving accurate measurement distance, some studies ${ }^{[29-31]}$ applied the Adaptive Kalman Filter (AKF) to processed the data, and illustrated the AKF can effectively restrain the filter divergence and has excellent filtering performance.

Thus, in order to achieve high-precision detection of crop heights in terms of light weight, this paper developed a crop height detection system based on a MWR, and a corresponding data fusion algorithm based on the AKF was developed to achieve accurate measurement of the crops height.

\section{Materials and methods}

\subsection{Hardware}

Figure 1 showed the types of altitudes during the UAV operation. This paper focused on the altitude of test object, due to its great significance for plant protection. Therefore, a system for detecting crop altitude was developed, as showed in Figure 2, consisting of four modules: a data acquisition module, a slave computer, a wireless transmission module and a host computer. DJI Phantom 3 (DJI Co, Ltd, Guangdong, China) was used to carry the modules.

In terms of the data acquisition module, an NRA24 MWR (Nanoradar Technology Co, Ltd. Hunan, China) was utilized to measure the relative altitude. An IMU, JY901 (Junyue Intelligent Control Technology Co, Ltd, Guangdong, China), was applied to measure flight angles and acceleration. Meanwhile, a global 
positioning unit, ATK-S1216F8-BD (SkyTraq Technology, Inc. Taiwan, China), was employed to get real-time positions of the UAV. OFor the wireless transmission module, digital transmission radio, XROCK V3 (Xili innovation Electronic Technology Co, Ltd, Zhejiang, China), was selected due to its long transmission distance. In addition, a microcontroller, STM32F103 (Xingyi Electronic Technology Co, Ltd, Guangdong, China), was exploited as the slave computer, while the host computer was a notebook computer. The specifications were listed in Table 1.

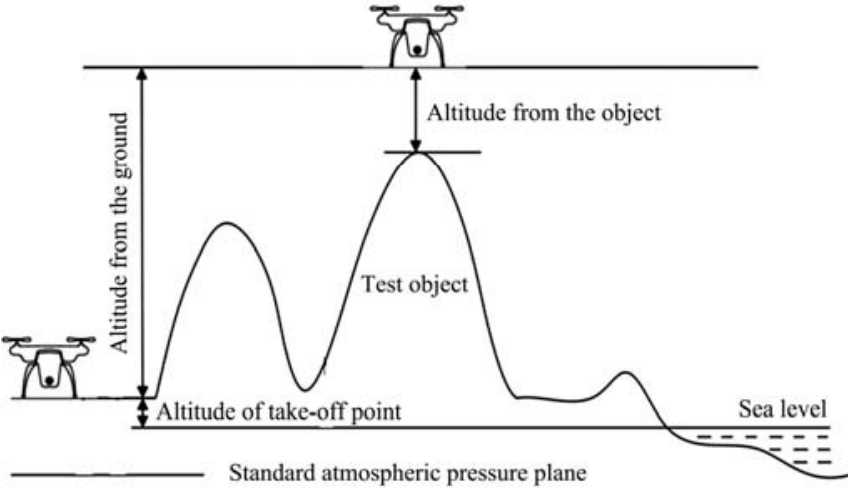

Figure 1 Definition of flight altitude of plant protection UAV

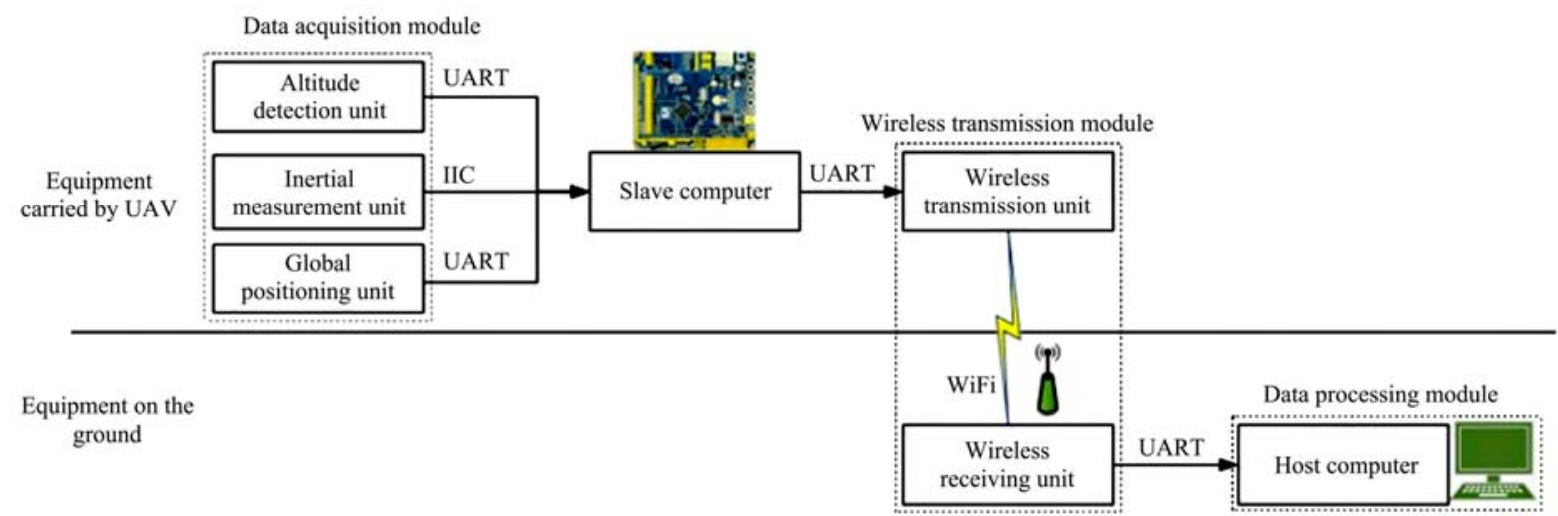

IIC: Inter-Integrated Circuit UART: Universal Asynchronous Receiver/Transmitter

Figure 2 Structure diagram of the detection system

Table 1 Specifications of NRB24 MWR, JY901 IMU, ATK-S1216F8-BD, XROCK V3, DJI Phantom 3

\begin{tabular}{|c|c|c|c|}
\hline Devices & Main parameter & Values & Remarks \\
\hline \multirow{5}{*}{ NRA24 MWR } & Transmitting frequency/GHz & $24.00-24.20$ & \\
\hline & Ranging distance $/ \mathrm{m}$ & $0.1-50$ & \\
\hline & Ranging accuracy $/ \mathrm{m}$ & \pm 0.02 & \\
\hline & Power consumption/W & $1.4-1.8$ & $5 \mathrm{~V} \mathrm{DC} 25^{\circ} \mathrm{C}$ \\
\hline & Size $/ \mathrm{mm}$ & $\begin{array}{c}130 \times 70 \times \\
14.5\end{array}$ & $\begin{array}{l}\text { Length } \times \text { Width } \\
\quad \times \text { Altitude }\end{array}$ \\
\hline \multirow{4}{*}{ JY901 IMU } & Operating Voltage/V & $3.3-5$ & \\
\hline & Operating current $/ \mathrm{mA}$ & 25 & \\
\hline & Measurement accuracy $/\left(^{\circ}\right)$ & 0.01 & \\
\hline & Size/mm & $\begin{array}{c}15.24 \times \\
15.24 \times 2\end{array}$ & $\begin{array}{l}\text { Length } \times \text { Width } \\
\times \text { Altitude }\end{array}$ \\
\hline \multirow{4}{*}{ ATK-S1216F8-BD } & Positioning accuracy/mCEP & 2.5 & \\
\hline & Update rate $/ \mathrm{Hz}$ & 20 & \\
\hline & Working temperature $/{ }^{\circ} \mathrm{C}$ & $-40-85$ & \\
\hline & $\mathrm{Size} / \mathrm{mm}$ & $25 \times 27$ & Length $\times$ Width \\
\hline \multirow{4}{*}{ XROCK V3 } & Baud rate & 57600 & \\
\hline & Communication distance $/ \mathrm{m}$ & $\leq 800$ & \\
\hline & Operating voltage/V & $3.7-6$ & $\mathrm{DC}$ \\
\hline & Size $/ \mathrm{mm}$ & $49 \times 25 \times 13$ & $\begin{array}{l}\text { Length } \times \text { Width } \\
\times \text { Altitude }\end{array}$ \\
\hline \multirow{4}{*}{ DJI Phantom 3} & Load/g & 1280 & \\
\hline & Working temperature $/{ }^{\circ} \mathrm{C}$ & $0-40$ & \\
\hline & Fight time/min & 23 & \\
\hline & Horizontal flight speed $/ \mathrm{m} \cdot \mathrm{s}^{-1}$ & $\leq 16$ & \\
\hline
\end{tabular}

2.2 Data fusion algorithm based on the Adaptive Kalman Filter (AKF)

The data fusion algorithm was based on the AKF. The data from the MWR, IMU and GPS was used to obtain the parameters of both the state equation and the measurement equation. Then, the data from the MWR were modified by the angle data from the IMU. Meanwhile, the modified data and the data from the GPS and IMU was asynchronously fused by the AKF to get final appropriate height results.

\subsubsection{Determination of the AKF}

The AKF was the key section for data fusion. The Figure 3 showed algorithm flow chart. According to the definition of the $\mathrm{AKF}$, the core is to determine the state equation and the measurement equation. These two equations in the flight condition were determined as follows.

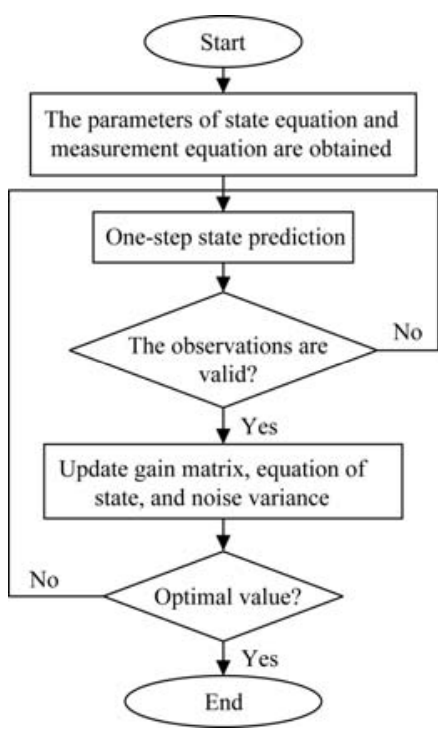

Figure 3 Algorithm flow chart

The data of the MWR and IMU was state variables. According to the relations of each state variables, the three basic aspects below could be obtained. 
1) If the flight altitude was $h_{r}$ and the noise generated by the MWR was $\omega_{1}$, the detection altitude by the MWR, $H_{m}$, was:

$$
H_{m}=h_{r}+\omega_{1}
$$

2) If the noise included in the position information by the GPS was $\omega_{2}$, and the crop height was $h_{f}$ and the detection altitude of the GPS, $H_{G P S}$, was:

$$
H_{G P S}=h_{f}+\omega_{2}+h_{r}
$$

$3)$ If the noise of the vertical acceleration by the IMU was $\omega_{3}$ and the actual vertical acceleration was $a_{r}$, the detection acceleration of the IMU, $a_{j}$, was:

$$
a_{j}=a_{r}+\omega_{3}
$$

Then, based on the definition of measurement equation, the measurement equation of the system should be:

$$
\left(\begin{array}{c}
H_{m} \\
H_{G P S} \\
a_{j}
\end{array}\right)=\left(\begin{array}{lll}
1 & 0 & 0 \\
1 & 0 & 0 \\
0 & 0 & 1
\end{array}\right) \times\left(\begin{array}{c}
h_{r} \\
v_{g} \\
a_{r}
\end{array}\right)+\left(\begin{array}{c}
\omega_{1} \\
\omega_{2} \\
\omega_{3}
\end{array}\right)+\left(\begin{array}{l}
0 \\
1 \\
0
\end{array}\right) \times h_{f}
$$

Meanwhile, the flight altitude, $h_{r}$, vertical acceleration, $a_{r}$, and velocity, $v_{g}$, were selected as state variables. If the UAV was uniform motion, the acceleration, $a_{j}$, will not change. Thus, according to the definition of the state equation, if the predictive value of acceleration was $\tau$, the state equation of the system, ( $h_{R}, a_{R}$, $\left.v_{G}\right)$, should be:

$$
\left(\begin{array}{l}
h_{R} \\
a_{R} \\
v_{G}
\end{array}\right)=\left(\begin{array}{lll}
1 & 0 & 0 \\
0 & 0 & 0 \\
0 & 0 & 1
\end{array}\right) \times\left(\begin{array}{l}
h_{r} \\
a_{r} \\
v_{g}
\end{array}\right)+\left(\begin{array}{l}
0 \\
1 \\
0
\end{array}\right) \times \tau
$$

Equation (4) and (5) were required to be respectively dispersed for the AKF.

In terms of the measurement equation, the discrete format was:

$$
W_{k}=A_{k} Y_{k}+Q_{k}
$$

For the state equation, the discrete format was:

$$
Y_{k}=\lambda_{k, k-1} Y_{k-1}+\xi_{k-1} G_{k-1}
$$

where, $Y_{k}$ was the state vector sequence; $W_{k}$ was the measurement sequence; $G_{k-1}$ and $Q_{k}$ were the white noise with zero mean; $\lambda_{k, k-1}$ was the state transition matrix; $\xi_{k-1}$ was the system noise driving matrix and $A_{k}$ was the measurement matrix.

In addition, Equation (6) and (7) must satisfy the following conditions:

$$
\begin{aligned}
& E\left\{G_{k}\right\}=E\left\{Q_{k}\right\}=0, E\left\{Q_{k} G_{k}\right\}=0 \\
& E\left\{G_{k} G_{j}^{T}\right\}=H_{k} \sigma_{k j}, E\left\{G_{k} G_{j}^{T}\right\}=U_{k} \sigma_{k j}
\end{aligned}
$$

where, $H_{k}$ was a nonnegative definite matrix; $U_{k}$ was a positive definite matrix; $\sigma_{k j}$ was Kroneker function, $\sigma_{k j}=1$, when $k$ is not equal to $j$.

If the flight time of the UAV is $t$, then the state transition matrix, $\lambda_{k, k-1}$, the system noise driving matrix, $\xi_{k-1}$, and the measurement matrix, $A_{k}$ can be obtained by Laplace transform of Equation (6) and (7). The results should be:

$$
\lambda_{k, k-1}=\left(\begin{array}{ccc}
1 & t & \frac{t^{2}}{4} \\
0 & 1 & t \\
0 & 0 & 1
\end{array}\right), \quad \xi_{k-1}=\left(\begin{array}{cc}
\frac{t^{3}}{6} & 0 \\
\frac{t^{2}}{2} & 0 \\
t & t
\end{array}\right), A_{k}=\left(\begin{array}{ccc}
1 & 0 & 0 \\
0 & 1 & 0 \\
1 & 0 & 0
\end{array}\right)
$$

Therefore, the temporal values of the elements in both the state vector sequence, $Y_{k}$, and the measurement sequence, $W_{k}$, can be determined, which will be the basis of the AKF in the temporal dimension. According to the equation (6) and (7), One-step prediction equation can be obtained:

1) If the optimal estimation of $k-1$ time, $Y_{k-1}$, and the priori estimate of $k$ time was $Y_{k / k-1}$, the one-step state prediction equation should be:

$$
Y_{k / k-1}=\lambda_{k, k-1} Y_{k-1}
$$

2) Assuming the covariance matrix of prior estimate of $k-1$ time was $P_{k-1}$, and the covariance matrix of optimal estimation of $k$ time was $P_{k / k-1}$, and the process noise matrix was $Z_{k-1}$, the one-step prediction mean square error matrix should be:

$$
P_{k / k-1}=\lambda_{k, k-1} P_{k-1} \lambda^{T}{ }_{k, k-1}+Z_{k-1}
$$

Then, based on the equation (10) and (11), the estimating equation can be obtained. Assuming the forgetting factor is $b$, the weighting coefficient, $d_{k}$ :

$$
d_{k}=(1-b) /\left(1-b^{k}\right)
$$

According to the equation (6) and (7), if the residual was $v_{k}$, so the residual calculation equation should be:

$$
v_{k}=W_{k}-A_{k} Y_{k-1}
$$

Based on the equation (12) and (13), the estimated measurement noise covariance, $R_{k}$, should be:

$$
R_{k}=\left(1-d_{k}\right) R_{k-1}+d_{k}\left(v_{k} v_{k}^{T}-A_{k} P_{k / k-1} A_{k}^{\mathrm{T}}\right)
$$

The equation (14), the estimated measurement noise covariance, $R_{k}$, have obtained, so the Kalman filter gain matrix, $K_{k}$, should be:

$$
K_{k}=P_{k / k-1} A_{k}^{T}\left(A_{k} P_{k / k-1} A_{k}^{T}+R_{k}\right)^{-1}
$$

According to the equation (13) and (15), the state estimation equation, $Y_{k}$ can be obtained:

$$
Y_{k}=K_{k / k-1}+K_{k} v_{k}
$$

Based on the equation (7) and (14), the estimated mean square error equation, $P_{k}$, should be:

$$
P_{k}=\left(1-K_{k} A_{k}\right) P_{k / k-1}
$$

Therefore, the one-step state prediction equation (10), the one-step prediction mean square error matrix (11), the Kalman filter gain matrix (15), the state estimation equation (16), and the estimated mean square error equation (17) have been obtained. The optimal estimation value of the altitude state can be gotten by using the above iterative equation.

\subsubsection{Asynchronous fusion of data}

In order to deal with the delay in data transmission, asynchronous fusion for the data from the MWR, IMU and GPS was developed. In a fusion cycle, according to Equation (6), (7), (15), (16) and (17), the minimum mean square error of the data from each sensor can be obtained by the AKF. Then, $\alpha$ and $\beta$ can be obtained, $\alpha$ was the $x$-axis offset angle and $\beta$ was the $y$-axis offset angle of the UAV. In order to increase the accuracy of the altitude data during the fusion, the altitude by the MWR, $H_{m}$, was modified based on flight angle. The modified altitude data, $H_{M}$, should be:

$$
H_{M}=H_{m} \cos \alpha \cos \beta
$$

Based on the equation (18), the covariance and Kalman filter gain matrix of the modified altitude data, $H_{M}$, and the data of the GPS can be obtained by the AKF. Meanwhile, the optimal updated value of each sensor can be determined and fused.

According to the data fusion and filtering based on the AKF, the altitude between the UAV and crop, $H_{\text {actual }}$, can be obtained. Assuming the altitude between UAV and ground was $H_{\text {relative }}$. Therefore, the height detection of crop, $H_{\text {crop }}$, should be:

$$
H_{\text {crop }}=H_{\text {relative }}-H_{\text {actual }}
$$

Then, the altitude data between UAV and crop by the MWR was $H_{\text {mactual }}$, which was raw data before the algorithm processing, and the crop height of the MWR, $H_{m c r o p}$, should be:

$$
H_{\text {mcrop }}=H_{\text {relative }}-H_{\text {mactual }}
$$

Based on the equation (19) and (20), the crop height after the algorithm processing, $H_{\text {crop }}$, and the crop height before the 
algorithm processing, $H_{\text {mcrop }}$, can be obtained.

\subsubsection{Simulation of the data fusion algorithm}

In order to indicate the accuracy of the algorithm, the simulation experiment of UAV flight altitude was established in MATLAB (MathWorks, Inc, America). Based on the pre-tests of the sensors during UAV flying, the measurement error of the MWR was set as $10 \mathrm{~cm}$ and that of the GPS was set as $0.5 \mathrm{~m}$. Meanwhile, the variance of actual acceleration was set as $0.01 \mathrm{~m}^{2} / \mathrm{s}^{4}$. The UAV was set as:

$$
h_{r}=200+10 \sin (0.1 t)
$$

Meanwhile, the relation between the velocity, and the flight time of the UAV, $t$, was:

$$
v_{g}=\cos (0.1 t)
$$

Moreover, the relation between the acceleration and the flight time of the UAV, $t$, was:

$$
a_{r}=-0.1 \sin (0.1 t)
$$

Figure 4 showed the error of altitude measurement by the simulation. It is obvious that although continually changing, the error was within $2 \mathrm{~cm}$, which indicated that the altitude data processed by the algorithm was closer to the actual value, so the data fusion algorithm was effective.

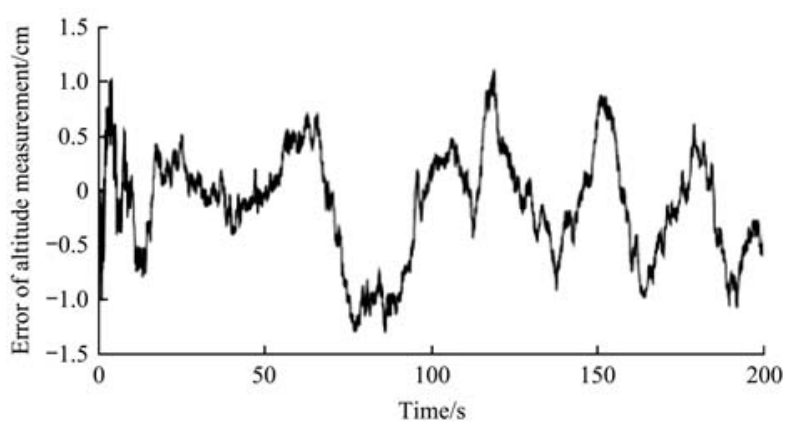

Figure 4 Error of altitude measurement

\subsection{Trial scheme}

\subsubsection{Test site and devices}

A corn field (Figure 5a) in Tongzhou, Beijing, was selected as the test site, with the corn at the seedling growth stage on April 25, 2019. Table 2 showed the devices and materials used in the tests, including a DJI Phantom 3 drone with a height detection system (Figure 5b), a computer and an altitude sign (Figure 5c). The altitude sign consisted of several $1 \mathrm{~m}$ long components.

\section{Table 2 The devices and materials}

\begin{tabular}{lcc}
\hline \multicolumn{1}{c}{ Devices } & Model & Quantity \\
\hline UAV & DJI Phantom 3 & 1 \\
MWR & NRA24 & 1 \\
IMU & JY901 & 1 \\
Global Positioning Unit & ATK-S1216F8-BD & 1 \\
Microcontroller & STM32F103 & 1 \\
Digital Transmission Radio & XROCK V3 & 1 \\
Altitude Sign & & 15 \\
\hline
\end{tabular}

\subsubsection{Trial design}

To verify the detection accuracy of the system, the height detection of corn trials was conducted. In order to ensure the accuracy of the trial, each group of the test was conducted three times. The following paces were implemented:

The DJI Phantom 3 was equipped with the height detection system. In Figure 6. Two flight altitudes from the ground, $5 \mathrm{~m}$ and $10 \mathrm{~m}$, were set. Altitude was determined by the UAV control system and the altitude sign. In addition, the UAV flew slowly and steadily on the corn plants for 3 minutes. In the trial, 15 sample points on the UAV flight path were selected. Manually measuring the corn height of each sample point to obtain the true height, $H_{t}$. The altitude between UAV and ground was $H_{\text {relative }}$.

Therefore, comparing the true value, $H_{t}$, the height from the system, $H_{\text {corn }}$, and the height from the MWR, $H_{\text {mcorn }}$, were compared to verify the accuracy of the system of height measurement.

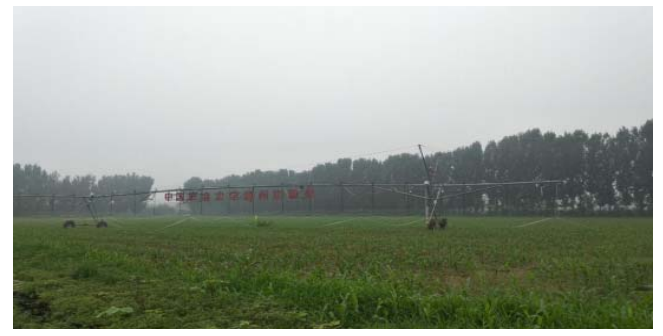

a. Test field

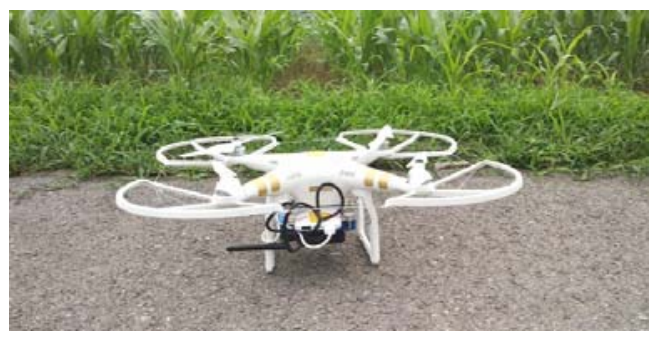

b. The height detection system and UAV

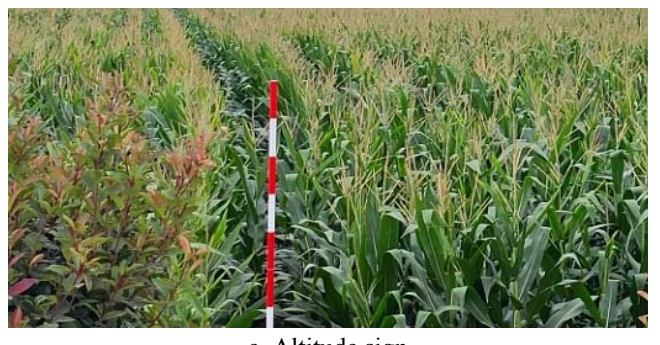

c. Altitude sign

Figure 5 Test site and devices

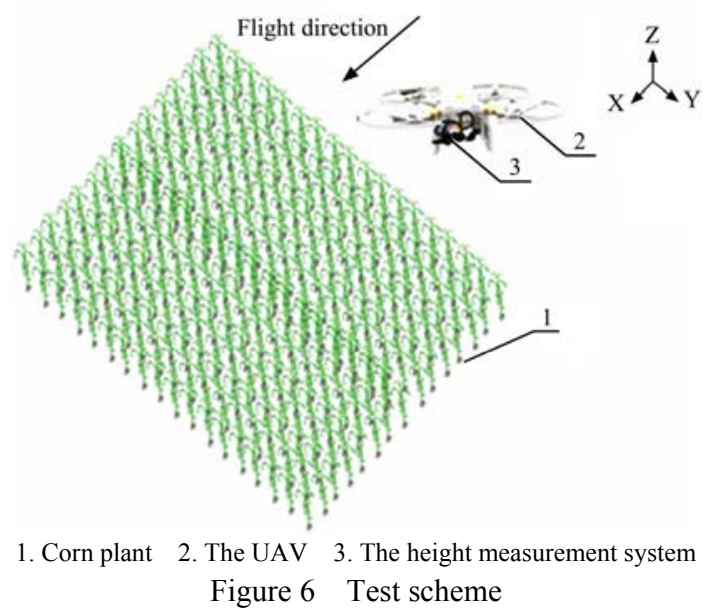

\section{Results and discussion}

\subsection{Analysis of accuracy}

The data from the MWR, the IMU and the GPS was processed by the algorithm based on the AKF. Figure 7 and Figure 8 show the variation of the altitude measured by the system.

The blue scatter line was the non-processed data, which was the raw data of the MWR, while the red solid line was the processed data. It was showed that the data was smoother after processing and sharp points were weakened. According to the two curves, the algorithm had removed the fluctuating noise of 
each sensor and the high distortion. For example, as showed in Figure 8, for the horizontal distance between 1600 and $1800 \mathrm{~cm}$, there were three data with severe distortion. When the flight altitude was at $10 \mathrm{~m}$, the noise of each sensor was eliminated. Therefore, the error had been significantly improved. The data of

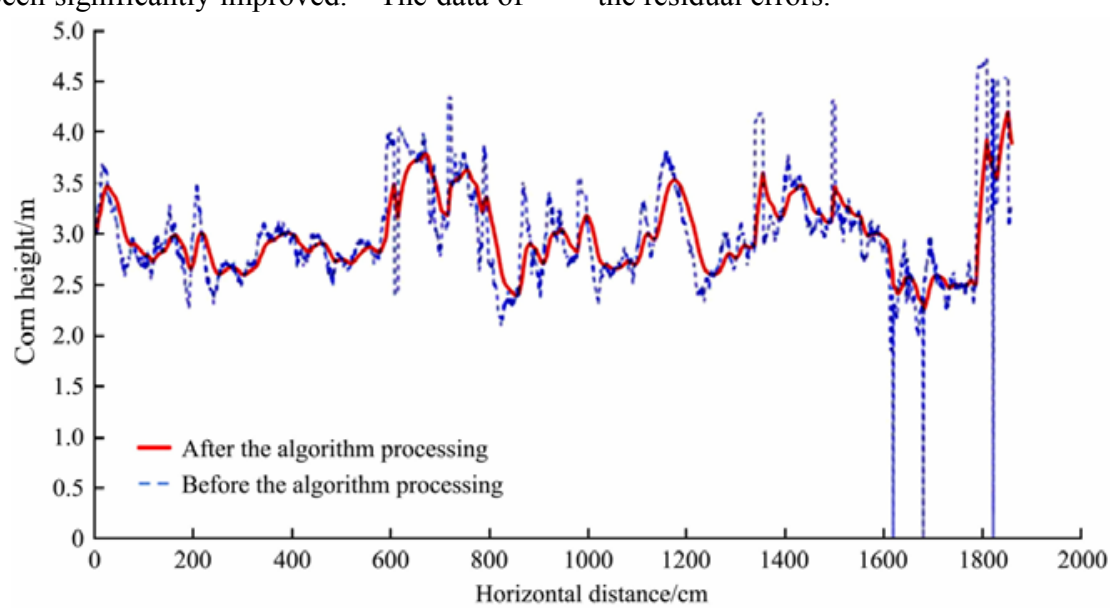

Figure 7 The corn height detected when the UAV flying at $5 \mathrm{~m}$ from the ground

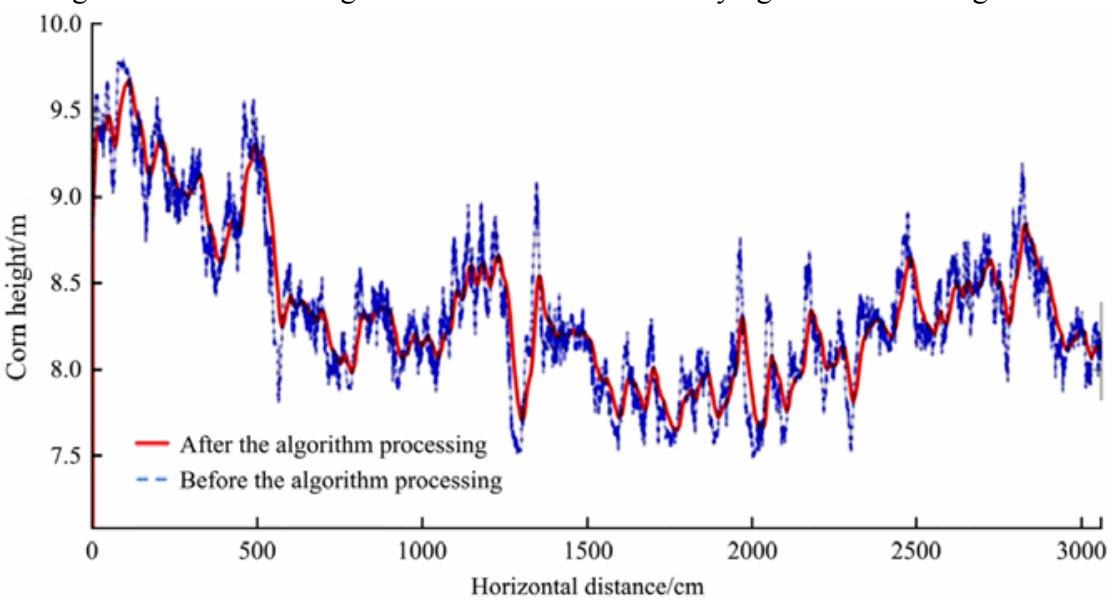

Figure 8 The corn altitude detected when the UAV was at $10 \mathrm{~m}$ from the ground

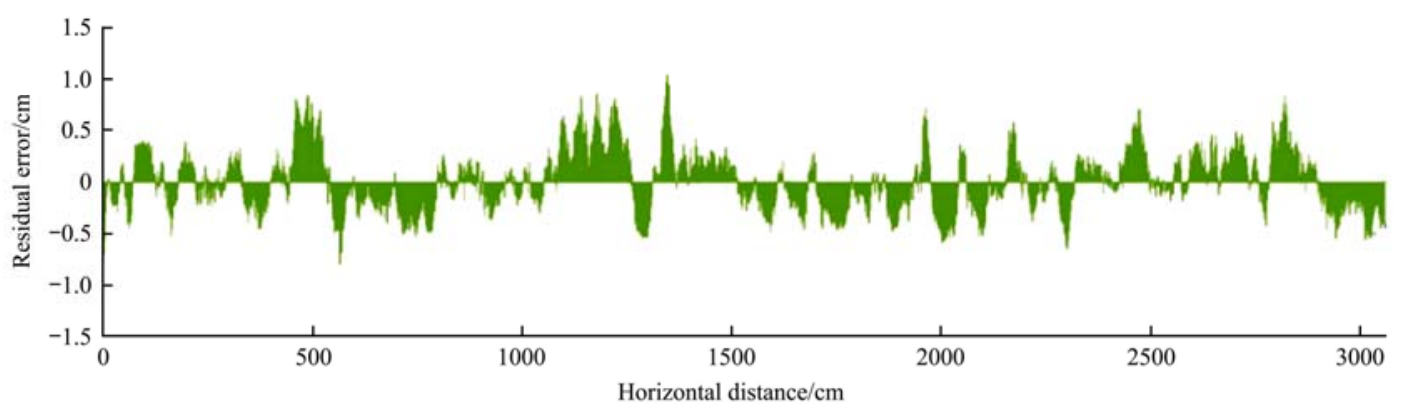

Figure 9 The residual errors of data processed by the algorithm

For the data processed by the algorithm, it showed that the residual error was concentrated within $\pm 1 \mathrm{~cm}$, close to zero. It indicated that the error of the processed data was significantly reduced. The degree of fitting was high, and it was more in line with actual situations. As showed in Table 3, the corn height value was took the average value when the flight altitude at $5 \mathrm{~m}$ and $10 \mathrm{~m}$ from the ground, and compare with the true corn height, $H_{t}$, the error value can be obtained.

The data processed by the algorithm, $H_{\text {corn }}$, was compared with the true crop height, $H_{t}$, and the error was maintained at $0.018 \mathrm{~m}$. The raw data, $H_{m c o r n}$, compared with the true crop height, $H_{t}$, the error was $0.053 \mathrm{~m}$. The error of the data processed by the algorithm was reduced by $0.035 \mathrm{~m}$ compared with the data before
15 sample points was fitted by the polynomial fitting model in MATLAB (MathWorks, Inc, America), which can accurately compare the errors before and after utilizing the algorithm. The flight altitude of $10 \mathrm{~m}$ was taken as an example. Figures 9 showed the residual errors. 
effectively minimise the noise.

Table 3 The corn heights before and after the algorithm processing

\begin{tabular}{ccccc}
\hline $\begin{array}{c}\text { True corn } \\
\text { height, } \\
H_{t} / \mathrm{m}\end{array}$ & $\begin{array}{c}\text { Corn height } \\
\text { before algorithm } \\
\text { processing, } \\
H_{\text {mcorn }} / \mathrm{m}\end{array}$ & $\begin{array}{c}\text { Error between } \\
H_{t} \text { and } H_{\text {mcorn }} \\
/ \mathrm{m}\end{array}$ & $\begin{array}{c}\text { Corn height } \\
\text { after algorithm } \\
\text { processing, } \\
H_{\text {corn }} / \mathrm{m}\end{array}$ & $\begin{array}{c}\text { Error between } \\
H_{t} \text { and } H_{\text {corn }} \\
/ \mathrm{m}\end{array}$ \\
\hline 0.620 & 0.536 & 0.064 & 0.614 & 0.006 \\
0.620 & 0.675 & 0.655 & 0.610 & 0.010 \\
0.880 & 0.922 & 0.042 & 0.868 & 0.002 \\
1.130 & 1.188 & 0.058 & 1.116 & 0.004 \\
1.000 & 0.902 & 0.098 & 1.025 & 0.025 \\
1.280 & 1.425 & 0.145 & 1.255 & 0.025 \\
1.630 & 1.664 & 0.034 & 1.648 & 0.018 \\
1.920 & 1.947 & 0.027 & 1.899 & 0.021 \\
1.720 & 1.647 & 0.073 & 1.694 & 0.026 \\
1.800 & 1.877 & 0.077 & 1.836 & 0.036 \\
1.800 & 1.769 & 0.031 & 1.823 & 0.023 \\
1.500 & 1.456 & 0.044 & 1.478 & 0.022 \\
1.640 & 1.665 & 0.025 & 1.650 & 0.010 \\
1.840 & 1.768 & 0.072 & 1.825 & 0.015 \\
1.810 & 1.797 & 0.013 & 1.794 & 0.016 \\
\hline
\end{tabular}

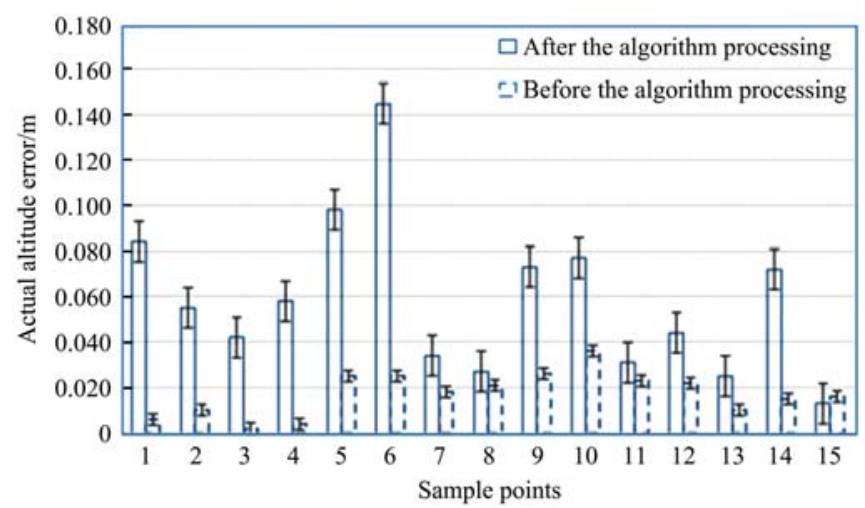

Figure 10 The errors of corn height

\subsection{Discussion}

In this paper, the theory research and system design of crop altitude accurate detection system based on the AKF was carried out. According to improve the height detection algorithm, the noise of the sensors was filtered. In order to deal with the delay in data transmission, asynchronous fusion for the data from the MWR, IMU and GPS was developed. The data error from the unstable flight of UAV was corrected by the flight angle from the IMU. The results showed that the crop height detection system have a high measurement accuracy. The system of crop height detection of UAVs obtain the data and store in the computer. In the seedling stage, the crop height data can be stored and sorted out by building a database. Meanwhile, the database can be used to analyse the growth process of crops and the utilization rate of pesticides and yield growth of crops have a greatly improve and reform.

The core of crop height detection system is the detection of UAV flight altitude. The current commercial spray drones are generally driven by manual operation, and the flying altitude of the UAV cannot be accurately detected in real-time. In the next stage, the altitude between the UAV and the crops can be connected with the UAV flight control system. Terrain-mimetic flight of the UAVs can be realized, and the utilization rate of pesticides will be improved.

\section{Conclusions}

This paper developed a crop height detection system based on the MWR and corresponding data fusion algorithm based on the AKF was proposed. Simulation tests and field trials were respectively conducted. The conclusions are:

1) Simulation test illustrated that the error of height was within $2 \mathrm{~cm}$ and less than $10 \mathrm{~cm}$, the purpose of system design was achieved, which demonstrated the feasibility of the system.

2) Field trials identified that compared with the raw data by the MWR, the error of detection system was reduced by $0.035 \mathrm{~m}$. Compared with real crop heights, the error of detection system was $0.02 \mathrm{~m}$. The developed system could achieve the accurate detection of the crop height. The system and algorithm provide a new theoretical model and technical idea for the UAVs configured for plant-protection.

\section{Acknowledgments}

This study was supported and financed by the National Key R\&D Program of China (No. 2016YFD0200702) and the National Key R\&D Program of China (No. 2018YFD0700603). The authors really appreciate the reviewers give their comments for the paper.

\section{[References]}

[1] Han W T, Li M, Chen W. Methods of Image Acquisition and Analysis for Crop Conditions Diagnose. Journal of Agricultural Mechanization Research, 2012, 34(006): 1-6.

[2] Huang Y Q, Huang T S, Yang R, et al. Detection and Test of Sugarcane Cutting Height Based on Machine Vision. Journal of Chinese Agricultural Mechanization, 2017(9): 81-87.

[3] Chen B Q, He C, Ma Y P, et al. 3D Image Monitoring and Modeling for Corn Plants Growth in Field Condition. Transactions of the Chinese Society of Agricultural Engineering, 2011, 27(13): 366-372. DOI: 10.3969/j.issn. 1002-6819.2011.z1.070.

[4] Zhang H C, Zhou H P, Zheng J Q, et al. Research Progress and Prospect in Plant Phenotyping Platform and Image Analysis Technology. Transactions of the Chinese Society for Agricultural Machinery, 2020, 051(003): 1-17.

[5] Fu H Y, Cui D D, Cui G X, et al. Progress in Crop Image Acquisition and Processing Techniques and Their Applications. Plant Fiber Sciences in China, 2019, v41; No.199(05): 40-50. DOI: CNKI:SUN:ZGMZ.0.201905-009.

[6] Sun Y Y, Shen S H. Research Progress in Application of Crop Growth Models. Chinese Journal of Agrometeorology, 40(07): 444-459.

[7] Wu L, Bo J H, Xiao Q, et al. Research Progress and Prospect on Combining Crop Growth Models with Parameters Derived from Quantitative Remote Sensing. Transactions of the Chinese Society of Agricultural Engineering, 2017, 33(009): 155-166. DOI: 10.11975/ j.issn.1002-6819.2017.09.020.

[8] Shao G M, Wang Y J, Han W T. Estimation Method of Leaf Area Index for Summer Maize Using UAV-Based Multispectral Remote Sensing. Smart Agriculture, 2020, v.2; No.7(03): 126-136.

[9] Zheng Y J; Yang S H; Zhao C J, et al. Modelling Operation Parameters of UAV on Spray Effects at Different Growth Stages of Corns. International Journal of Agricultural and Biological Engineering, 2017.05, 10(3): 57-66. DOI: 10.3965/j.ijabe.20171003.2578.

[10] Zheng Z, Yang S H, Zheng Y J, et al. Obstacle Avoidance Path Planning Algorithm for Multi-rotor UAVs. Transactions of the Chinese Society of Agricultural Engineering, 2020, 36(23): 59-69.

[11] Papa U, Core G D. Design of Sonar Sensor Model for Safe Landing of an UAV [C] Metrology for Aerospace, Benevento, Italy. IEEE, 2015.

[12] Wen T, Gao S, Zou H C. Based on Laser Ranging Method Research of UAV Terrain Matching Flight. Computer Measurement \& Control, 2015, 23(9): 3209-3212.

[13] Wang S L, Li D J, Fan Y, et al. Design of Laser Ranging Sensor for Short Distance Measurement. Foreign Electronic Measurement Technology, 2016, 35(11): 98-102. 
[14] Huang K L, Chiu C C, Chiu S Y et al. Monocular Vision System for Fixed Altitude Flight of Unmanned Aerial Vehicles. Sensors (Basel, Switzerland), 2015: 16848-16865. DOI: 10.3390/s150716848.

[15] Mogyla A, Khlopov G. Measurement of Range and Speed of High-Speed Targets by Millimeter Wave Pulse Radar. 2016 ninth International Kharkiv Symposium on Physics and Engineering of Microwaves, Millimeter and Submillimeter Waves (MSMW). IEEE, 2016. DOI: 10.1109/MSMW.2016.7538164.

[16] Caris M, Stanko S, Johannes W, et al. Detection and Tracking of Micro Aerial Vehicles with Millimeter Wave Radar. Radar Conference. London, UK. IEEE, 2017.

[17] Wu L, Peng S S, Xiao Z L, et al. Method for Improving Ranging Accuracy of Short-range Millimeter Wave Radar. Acta Arma, 2009, 30(03): 301-305.

[18] Tian H W, Xu Y L, Sheng X F. Principle Analysis and Simulation of Height Measurement by Plant Protection UAV Millimeter-wave Radar. Modern Electronics Technique, 2020, v.43; No.556(05): 175-178. DOI: CNKI:SUN:XDDJ.0.2020-05-040.

[19] Zhou F, Tian H B, Ysng H. High-Precision Radar Altimeter Design for Small UAV. Chinese Journal of Electron Devices, 2019: 1179-1184.

[20] Wang Hui. Research on Accurate Altitude Measurement and Obstacle Avoidance Strategies of Quadcopter UAV. Sichuan: China West Normal University, 2020.

[21] Ling F, Shi T S, Lu Y J, et al. Design and Implementation of UAV's Terrain Following System Based on Lidar. Fire Control \& Command Control, 2020, 45(09): 146-151, 156.

[22] Geng K K, Chulin N A. Applications of Multi-Height Sensors Data Fusion and Fault-Tolerant Kalman Filter in Integrated Navigation System of UAV. Procedia Computer Science, 2017, 103: 231-238. DOI: 10.1016/j.procs.2017.01.090.
[23] Song Y, Wen X W, Guo X G. Small UAV Localization Method Based on Optical-Flow and Inertial Navigation. Transducer and Microsystem Technologies, 2015, 34(1): 13-16.

[24] Campos I, Nasimento E, Freitas G, et al. A Height Estimation Approach for Terrain Following Flights from Monocular Vision. Sensors, 2016, 16(12): 2071. DOI: 10.3390/s16122071.

[25] Huang H, Liu Y H, Zhao X. Multi-Layer Multi-Source Information Fusion Algorithm for Hovering UAV Height Measurement. Journal of Chinese Inertial Technology, 2018, 026(003): 316-322, 329. DOI: 10.13695/j.cnki.12-1222/o3.2018.03.007.

[26] Tu W J, Shen Q H, Wang X M. Estimating UAV Altitude Based on Kalman Filter. Journal of Nanchang Hangkong University (Natural Sciences), 2019: 101-104. DOI: CNKI:SUN:NCHK.0.2019-04-017.

[27] $\mathrm{Hu} \mathrm{D}$ D, Gu Y, Gao Q J. Precise Altitude Measuring Method of Flight-Inspecting UAV Based on Fault-Tolerant Kalman Filter. Journal of Civil Aviation University of China, 2019, 037(003): 17-21.

[28] Wu K H, Sun X C, Zhang J C. Terrain Following Method of Plant Protection UAV Based on Altitude Fusion. Transactions of the Chinese Society for Agricultural Machinery, 2018, 49(6): 17-23.

[29] Zhou Q F, Li Z, Yu G Z, et al. A Novel Adaptive Kalman Filter for Euler-Angle-Based MEMS IMU/Magnetometer Attitude Estimation. Measurement Science and Technology, 2021, 32(4). DOI: 10.1088/ 1361-6501/abcefa.

[30] Jun L; Luo J; Fan Y K, et al.Vehicle Platform Attitude Estimation Method Based on Adaptive Kalman Filter and Sliding Window Least Squares. Measurement Science and Technology, 2021, 32(3). DOI 10.1088/1361-6501/abc5f8

[31] Chen Y, An Z D, Chen H, et al. A UWB Location Algorithm--Based on Adaptive Kalman Filter. Journal of Physics: Conference Series, 2021, 1757(1): 012176 (7pp). DOI: 10.1088/1742-6596/1757/1/012176. 